\title{
Multi-wavelength studies from optical to radio wavelengths
}

\author{
S. Pohjolainen ${ }^{1}$ \\ ${ }^{1}$ Tuorla Observatory/VISPA, University of Turku, FI-21500 Piikkiö, Finland email: \\ silpoh@utu.fi
}

\begin{abstract}
This paper reviews some recent results obtained from multi-wavelength studies that extend from white light to radio. Special emphasis is given to radio observations, which can be used to determine emission mechanisms and to trace electron propagation paths and plasma motions. Multi-wavelength comparisons have been found to be essential when analysing flareand CME-related eruptions, but significant results have also been obtained in 'quiet' Sun studies.
\end{abstract}

\section{Introduction}

Different densities and temperatures, and therefore different heights in the solar atmosphere can be probed with observations at different wavelengths. EUV images will show us the low-lying cooler loops and soft X-rays will tell us the locations of the hot loop tops, with magnetograms telling us the connectivities and field strengths, and radio observations giving a hint of the emission mechanisms and electron propagation paths. Radio emission is a strong diagnostic in determining whether the emission mechanism is thermal or non-thermal (at millimeter-centimeter wavelength range we can observe both bremsstrahlung and gyrosynchrotron emission), and at decimetric-metric wavelengths we can follow the electron propagation paths and coronal shocks by observing the subsequent plasma emission at the local plasma frequency in the surrounding medium.

Multi-wavelength observations become crucial when we try to analyse something with an eruptive nature. In flare eruptions we need to know which loops and loop systems are involved, how the region evolves before eruption, where the particles get accelerated and where they travel, and if some of the magnetic field lines are open or not. In CME studies we often see the whole repertoire of features and events which can be interpreted as shock signatures (Moreton waves in $\mathrm{H} \alpha$, EIT-waves in EUV, SXT-waves in soft X-rays, type II emission at low radio frequencies, filament oscillations in $\mathrm{H} \alpha$ ), depletion of matter (dimmings and disappearing loops in EUV and soft X-rays), moving magnetic structures (rising filaments visible in $\mathrm{H} \alpha$ and EUV, trapped electrons observed as type IV radio emission), and large-scale restructuring and opening of the field lines (radio emission tracing the paths of electron beams), just to mention some. To be able to determine onset times and lift-offs and to be able to correlate something visible in the chromosphere to something traveling in the interplanetary space we need a large wavelength coverage. Some examples and recent results from multi-wavelength studies that include radio observations are presented in this paper.

\section{The 'quiet' Sun from optical to radio wavelengths}

Radio emission at centimeter and millimeter wavelengths has been of interest for several decades because of it's complexity: coronal holes and polar regions sometimes show up in enhanced emission and sometimes in reduced emission, with some dependence on the 
wavelength. First studies (see summary in Kosugi, Ishiguro \& Shibasaki 1986) suggested that solar polar regions are radio bright in a certain wavelength range. The suggestion of uniformly radio bright polar coronal holes may have come from observations with poor spatial resolution - a real problem in radio astronomy when trying to image structures that have small intensity and/or are much wider than simple point sources. (The spatial resolution with a single dish antenna can be approximated with $\theta \approx 1.2 \lambda / D$, where $\lambda$ is the wavelength and $D$ antenna diameter, this shows how the long wavelengths in radio need to be imaged with large antennas - in practice good spatial resolution is obtained only with interferometers, in solar physics they are called radioheliograps.)

In a study using the Nobeyama Radioheliograph, with the best spatial resolution available at $17 \mathrm{GHz}(10$ arc sec, $\lambda=1.8 \mathrm{~cm})$, Shibasaki (1998) suggested that the diffuse, enhanced level of radio emission in the polar-cap region is due to limb brightening, with the bright patches superimposed on it. Nindos et al. (1999) suggested that the diffuse emission areas at polar caps could be associated with He II $304 \AA$ features - but no oneto-one correlation was found between the radio bright patches and the EUV structures. At higher frequencies, Pohjolainen et al. (2000) showed that the enhanced emission at 87 $\mathrm{GHz}(3.5 \mathrm{~mm})$ correlates with the locations of the bases of polar plumes in EUV, with bright points in X-rays and EUV, but also with dense diffuse structures in EUV and soft X-rays. Riehokainen et al. (2001) associated the radio brightenings also with polar faculae observed in white light. Recently, Selhorst et al. (2003) also associated radio limb brightening with faculae.

Polar radio brightenings in general are related to the question whether coronal holes are radio bright, or if there are local radio brightenings inside coronal holes. Multiwavelength comparisons of radio emission from equatorial coronal holes (Gopalswamy et al. $1998 ; 1999)$ showed that the radio enhancement inside coronal holes is not uniform, but inhomogeneous with large less-intense brightenings and small intense point-like brightenings, that are most probably caused by temperature enhancements in the chromosphere as no specific correlation was found with the structures observed at higher temperature EUV lines. Moran et al. (2001) then compared the $17 \mathrm{GHz}$ microwave-enhanced regions in equatorial coronal holes with EUV to UV wavelengths, in a search for temperature increases which might explain the bright spots at radio wavelengths. The co-spatiality of the $17 \mathrm{GHz}$ and $\mathrm{H} \alpha$ enhancements and the equal integrated normalized enhanced emission and brightness temperatures suggested that the radio enhancements were caused by increased fibril radio emission. There are still some unanswered questions in the formation of radio bright structures inside coronal holes, that wait for simultaneous multiwavelength observations with better spatial resolution.

\section{Small-scale events and flares}

Several X-ray-radio comparisons have been done from X-ray network flares (Krucker et al. 1997), which are more than an order of magnitude smaller than X-ray bright points or active region transient brightenings, to studies of larger flare events. Liu et al. (2004) studied microflares and concluded that their time profiles in soft and hard X-rays and in $\mathrm{H} \alpha$ show temporal evolution that resembles that of large flares. Co-aligned imaging observations showed that the events all occurred in active regions and were located near magnetic neutral lines. The findings suggest that some microflares are produced by magnetic reconnection, which results in closed compact loops and open field lines, which in their turn are indicated by the associated radio type III bursts.

A study of simple, short-duration radio flares suggested that they originated from interacting two loops, contrary to the previous assumption that they all apprear as single loop 


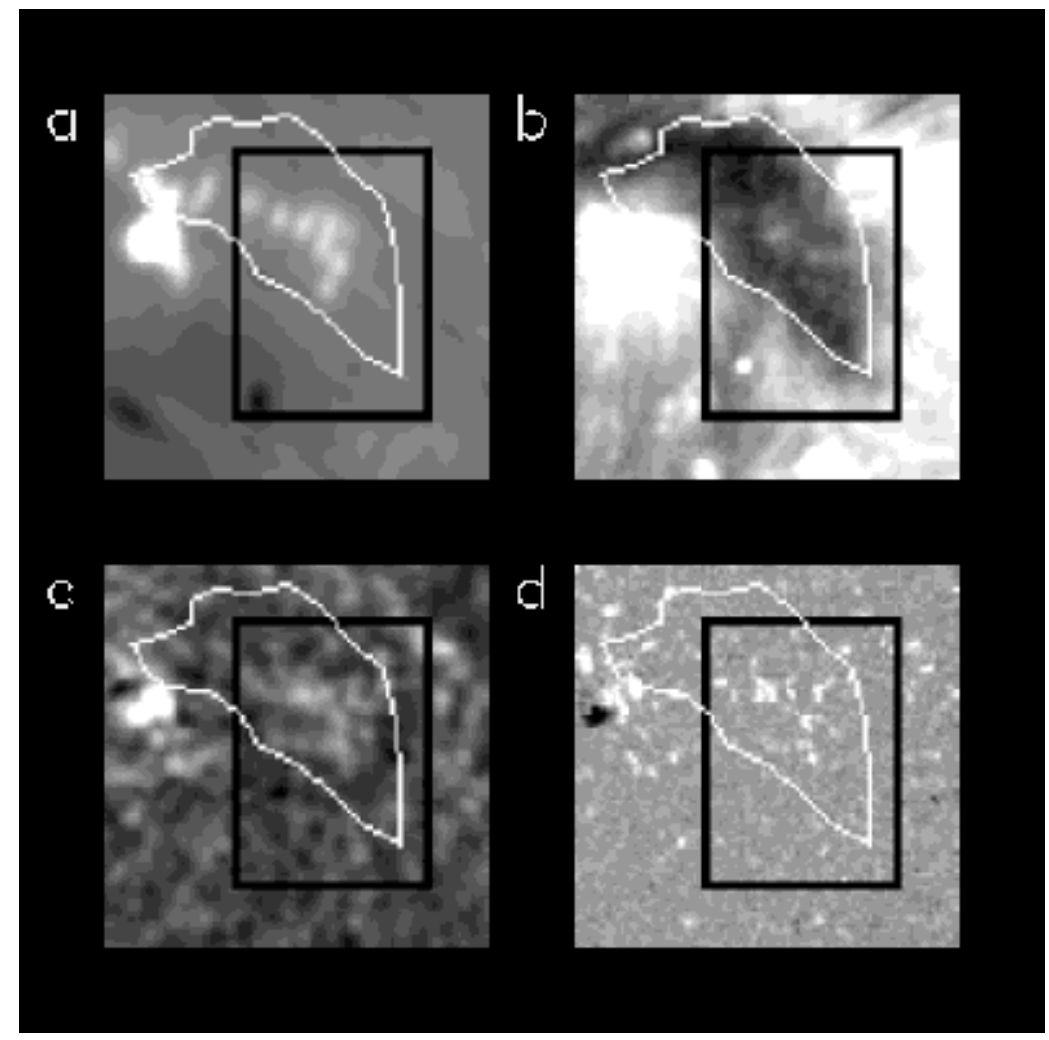

Figure 1. Looking for the cause for radio brightenings inside coronal holes: images of an equatorial coronal hole in a) $17 \mathrm{GHz}$ radio, b) $195 \AA$ (EIT Fe XII), c) $\mathrm{H}_{\alpha}$ emission, and d) the corresponding SOHO/MDI magnetogram. The coronal hole boundary is outlined in white and the SUMER field of view is outlined in black. $\mathrm{H}_{\alpha}$ emission shows best correlation with the point-like radio brightenings. (From Moran et al., Astron. Astrophys., 2001.)

events (Kundu et al. 2004a). Loop-loop interactions were also suggested by Pohjolainen (2003) to be the mechanism for repeated radio flaring. In that study it was essential to be able to identify the separate soft X-ray and EUV loop structures, in relation with the radio and hard $\mathrm{X}$-ray source locations.

Lee et al. (2003) studied a flare that lacked the separating motion of $\mathrm{H} \alpha$ ribbons. The microwave bursts had very short duration, in spite of the large amount of soft X-ray flux. This could be explained by the scena rio of a confined flare, since energy release via spontaneous reconnection in a confined magnetic structure can be very rapid. Recent X-ray observations from the newly launched RHESSI together with TRACE high spatial resolution EUV observations, and radio images at centimeter wavelengths (Nobeyama) have demonstrated the diversity of energy release and non-thermal acceleration sites (Kundu et al. 2004b). For example, in a multi-wavelength study by White et al. (2003), the preflare radio images show a large hot long-lived loop not visible at other wavelengths.

\section{Large scale events: CMEs and shocks}

A long-standing problem has been the origin of CMEs, and the details of their relationship to a number of associated phenomena like solar flares, coronal and interplanetary type II radio bursts, shocks, and solar energetic particle (SEP) events, to name a few. 


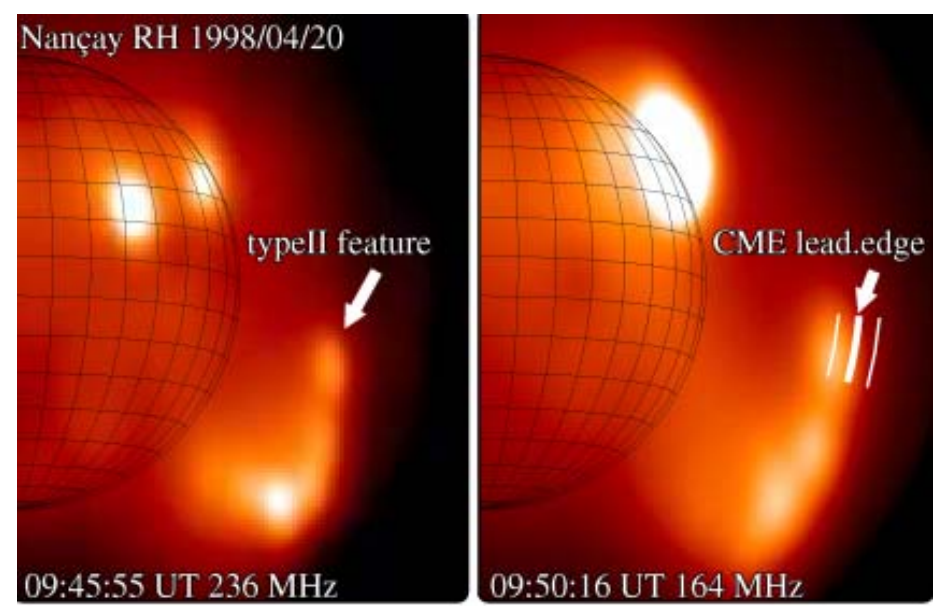

Figure 2. Nancay radio type II burst positions (on the left), superposed with the extrapolated CME leading edge (on the right). Nancay observations suggest emission at the fundamental and second harmonic. (Image courtesy of D. Maia)

Radio observations by themselves are an excellent way to probe large-scale plasma motions, either by the emission of the electrons themselves or by the surrounding medium. Radio studies have shown activated prominences that rotate around their vertical axis while moving on the solar surface, an activity which continues in the CME core and in the neighboring streamer (Hori 2000). They have shown 'CME cannibalism' where a fast CME overtakes a slower one, producing a radio enhancement during the 'swallowing' phase in the heliosphere, visible at long radio wavelengths (Gopalswamy et al. 2001). And they have produced the first direct radio images of expanding CME loops near $\mathrm{R}=3 \mathrm{R}_{\text {sun }}$ (Bastian et al. 2001). No counterpart to white-light CMEs had been observed at other wavelengths before.

CME fronts have been observed (Maia et al. 2000) to be the drivers of coronal type II emission, too - but here the association is based on comparisons to extrapolated CME locations in white light, which can be uncertain. Coronographs block the emission from the solar disk and CMEs are usually first detected far away from the solar limb, thus making direct comparisons of emission locations very difficult.

Gopalswamy et al. (1997) observed a type II burst at the leading edge of ejected Xray material that was moving with a speed of about $900 \mathrm{~km} \mathrm{~s}^{-1}$, while a type IV burst was associated with a detached plasmoid consisting of heated prominence material that followed the X-ray eruption. Klein et al. (1999) observed an ejected plasma blob, which upon arrival at the top of the pre-existing loops (i.e. a region of low Alfven speed) generated a shock wave that became visible as type II emission. Type II bursts above expanding soft X-ray loops have been reported also by Klassen et al. (1999;2003). The loop expansion speed in the later case was estimated to be around $700 \mathrm{~km} \mathrm{~s}^{-1}$.

Pohjolainen et al. (2001) reported spatially coinciding EIT and $\mathrm{H} \alpha$ (Moreton) wave fronts that were associated with unusually fast drifting type II-like bursts. The radio burst sources appeared in close temporal and spatial coincidence with the extrapolated location of the EUV $/ \mathrm{H} \alpha$ wave fronts. The $\mathrm{H} \alpha$ wave speed was $630-790 \mathrm{~km} \mathrm{~s}^{-1}$. Khan \& Aurass (2002) later compared the emission locations of a (typical) radio type II burst with a moving soft X-ray front, an EIT wave front, and an $\mathrm{H} \alpha$ (Moreton) wave front in the well-covered event on November 3, 1997. They found spatial correlation for all of these features. In two other papers correlation has been established between $\mathrm{H} \alpha$ and 

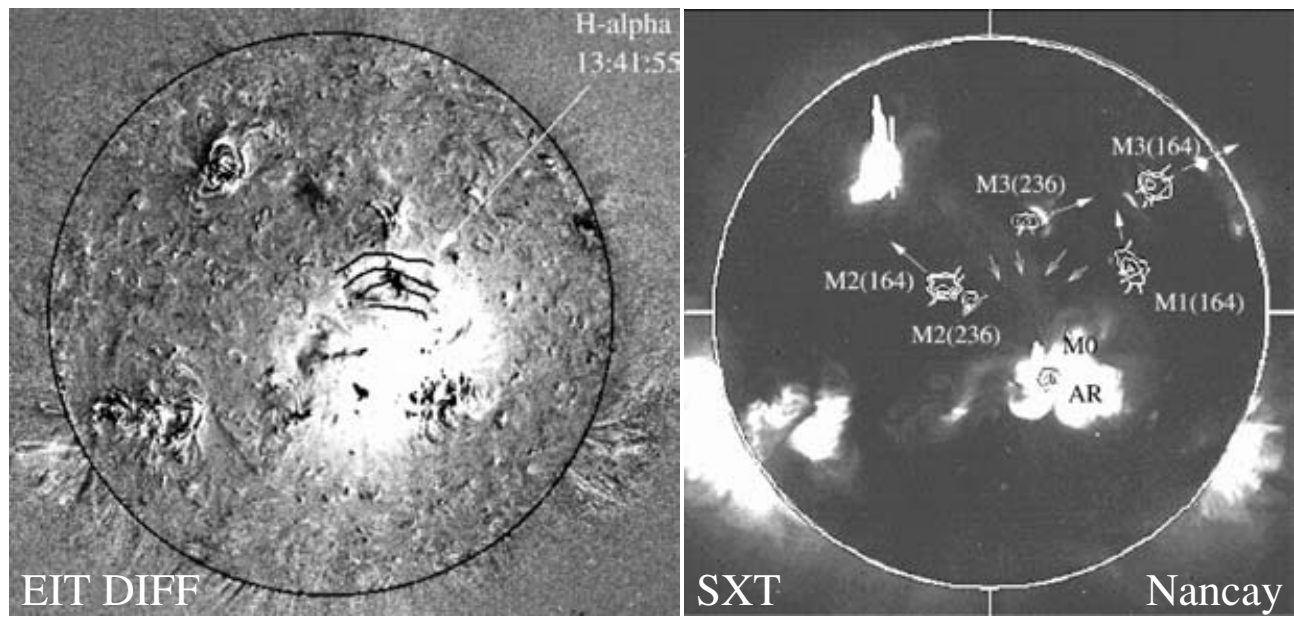

Figure 3. Left: Kanzelhöhe $\mathrm{H} \alpha$ wave fronts (contours) plotted over the SOHO EIT difference image on May 2, 1998. Right: Nancay radio type II-like burst sources M1-M3 (contours) plotted over a soft X-ray image (Yohkoh SXT). Arrows point to the location of the Moreton wave front observed about one minute before the start of M1. (Pohjolainen et al. Astrophys.J., 2001.)

soft X-ray wave fronts (Narukage et al. 2002) and between soft X-ray and an EUV wave fronts (Hudson et al. 2003). In both cases a type II burst was reported, but not imaged.

Despite (or because of) the divergent observations of type II burst drivers, Claßen \& Aurass (2002) have suggested that they might originate either from flare-related blast wave shocks or shocks driven by the leading edges, internal parts, or flanks of CMEs. There is observational evidence to support all the alternatives, but one should also take into account problems like projection effects, and error sources like the use of different density models in speed determination (see, e.g., Mancuso \& Raymond 2004).

\section{Early signatures of large scale changes}

Radio spectra can be used to find CME-related characteristics: Aurass et al. (1999) noted that in the case of a CME event type III burst groups appeared in the same time interval when first CME-related structural changes were observed, and frequency-drifting continuum emission (type IV) was seen when the CME matter passed the low corona (C1 field of view in LASCO). Khan \& Hudson (2000) first reported sudden disappearances of transequatorial loops in connection with CMEs, and soon radio emission in the form of J-bursts (a type III subclass) were discovered to trace the interconnecting loops just before large-scale changes and dimmings set on (Pohjolainen et al. 2001;2004).

There are also some special radio features associated with CME lift-offs: Klassen et al. (1999) pointed out the type II precursors and 'arc' features (inverted U-shaped spectral envelope) from the dynamic spectra, observed just before the start of type II bursts. They proceeded to compare the precursor locations with structures in soft X-ray images, and determined that the precursor and arc locations are above or near soft X-ray loop tops, in between the $\mathrm{H} \alpha$ and type II emission sites. Klassen et al. (2003) further suggested a close connection between radio precursor structures and hard X-ray sources - both signatures of electron acceleration. Another interesting CME-type II-precursor event has been studied by Lehtinen et al. (2004), who noticed precursor connection with decimetric reverse drift bursts, that have been associated with rising plasmoids and electron acceleration (Karlický 2004; Karlický et al. 2003). 


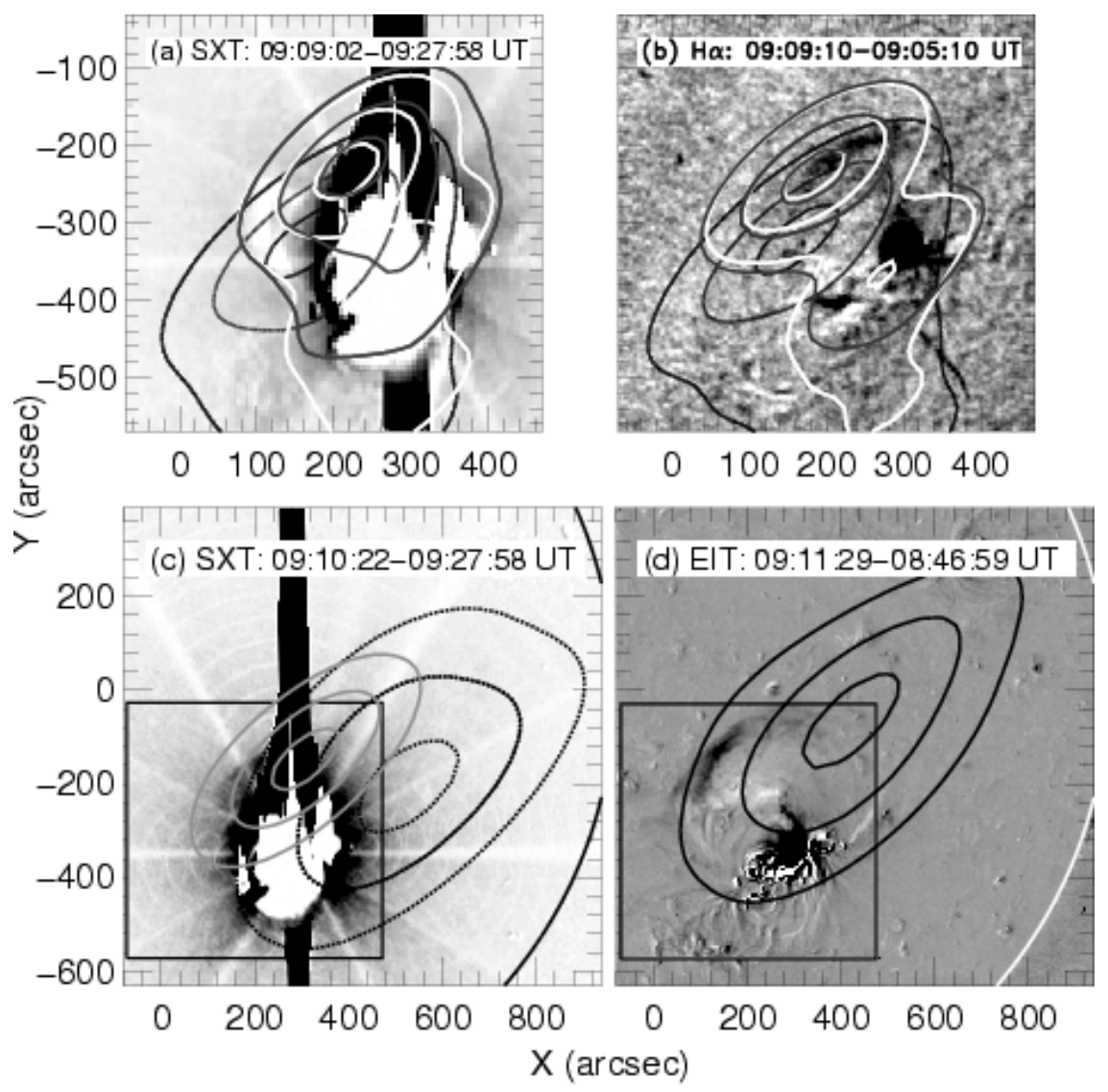

Figure 4. Portions of soft X-ray (Yohkoh SXT), H $\alpha$ (Kanzelhöhe), and EUV (SOHO EIT) difference images with overplotted Nancay Radioheliograph image contours during the type II burst emission, close to the first times indicated in each panel. The image shows how the type II burst emission is located near the soft X-ray, EIT, and Moreton wave fronts. (From Khan \& Aurass Astron.Astrophys., 2002.)

\section{From the solar corona to the heliosphere}

Coronal mass ejections travel a long way from the solar corona into the interplanetary space, and solar energetic particles (SEPs) get accelerated during this process. With multiwavelength observations we can follow the processes, structural changes, and the behavior of the magnetic field. In a recent study by Mandrini et al. (2004) a small active region (the size of an X-ray bright point) was shown to undergo an eruption, resulting in an EUV dimming and cusp, and which was later observed as a small magnetic cloud in the interplanetary space. Several SEP studies have used white light, EUV, X-ray, and radio data to determine where the particles get accelerated (e.g., Torsti et al. 2002; 2003).

To better understand the full evolution of solar events, and to model them, we will need coronal observations with better sensitivity (dynamic range) and better spatial resolution. There are several new satellite missions and developments in ground-based instruments under way that will improve the observational side in the future.

\section{Acknowledgements}

Multi-wavelength studies require a lot of data, and software to handle it all with. We would like to acknowledge the free-use data policy of many solar satellite missions and 

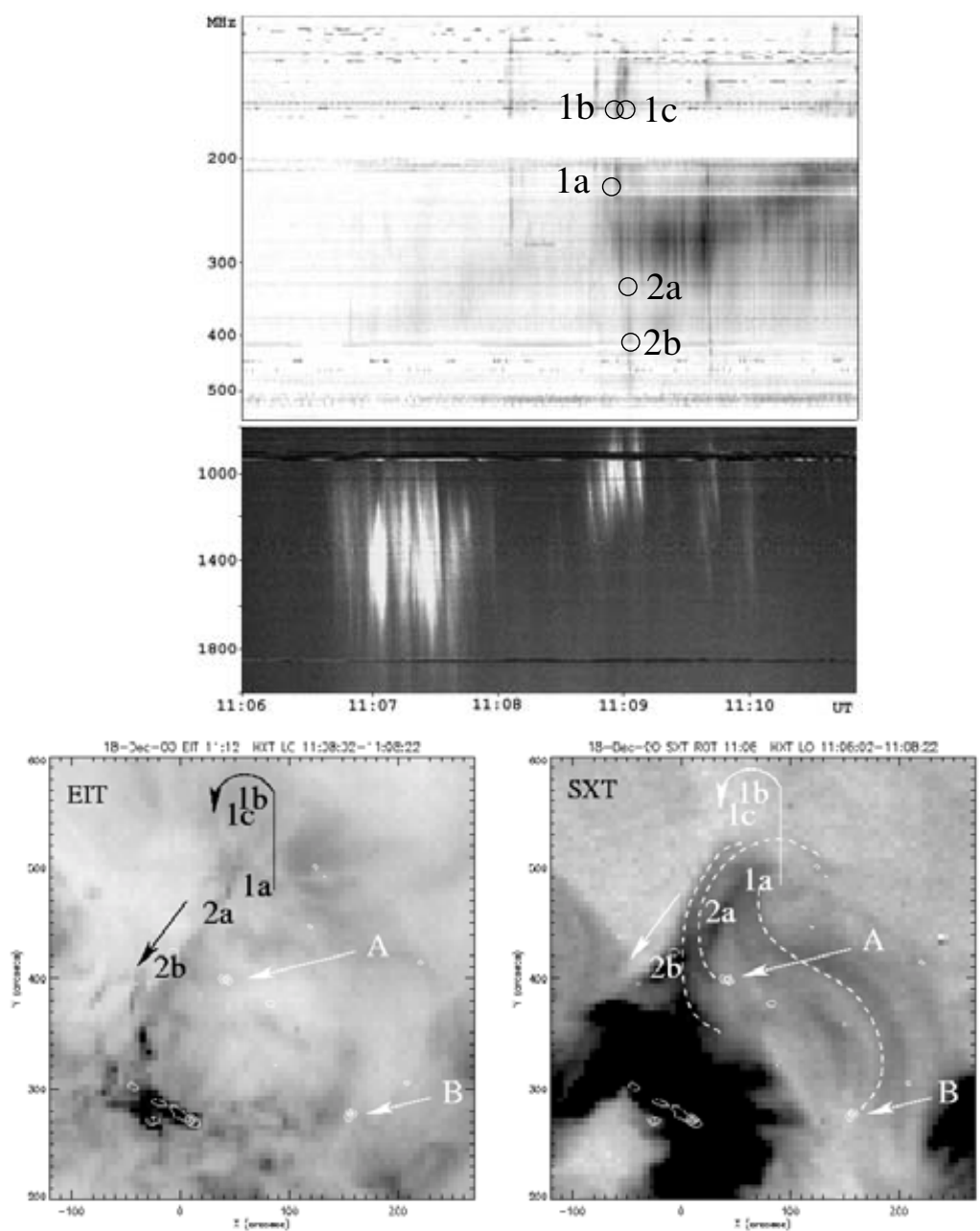

Figure 5. J-bursts and drifting decimetric features associated with a flare and a halo CME. One J-burst $(1 \mathrm{a}-1 \mathrm{c})$ and a following reverse drift-burst $(2 \mathrm{a}-2 \mathrm{~b})$ are marked on the frequency-drifting precursor envelope. The burst locations are plotted over SOHO EIT (bottom left) and Yohkoh SXT (bottom right) images. Association with hard X-ray sources ' $\mathrm{A}$ ' and ' $\mathrm{B}$ ' was also established. Dotted white lines show where some of the soft X-ray loops are located. (Analysis from Lehtinen et al. Astron.Astrophys., 2004, submitted)

ground-based observatories, and the effort they have put in creating and maintaining their internet data archives. The developers of the Solarsoft package are thanked for not loosing their nerves with us.

\section{References}

Aurass, H., Vourlidas, A., Andrews, M.D., Thompson, B.J., Howard, R.H., and Mann, G. 1999 Astrophys. J. 511, 451-465.

Bastian, T.S., Pick, M., Kerdraon, A., Maia, D., Vourlidas, A. 2001 Astrophys. J. 558, L65-69.

Claßen, H.T. \& Aurass, H. 2002 Astron. Astrophys. 384, 1098-1106.

Gopalswamy, N., Kundu, M.R., Manoharan, P.K., Raoult, A., Nitta, N., and Zarka, P. 1997 Astrophys. J. 486, 1036-1044.

Gopalswamy, N., Shibasaki, K., DeForest, C., Bromage B.J.I. and Del Zanna, G. 1998 In Synoptic 
Solar Physics (ed. K.S. Balasubramaniam, J. Harvey, and D. Rabin). ASP Conf. Ser. 140, 363-371.

Gopalswamy, N., Shibasaki, K., Thompson, B.J., Gurman, J., and DeForest, C. 1999 J. Geophys. Res. 104, 9767-9780.

Gopalswamy, N., Yashiro, S., Kaiser, M.L., Howard, R.A., Bougeret, J.-L. 2001 Astrophys. J. 548, L91-94

Hori, K. 2000 Astrophys. J. 543, 1011-1015.

Hudson, H.S., Khan, J.I., Lemen, J.R., Nitta, N.V., and Uchida, Y. 2003 Solar Phys. 212, 121-149.

Karlický, M. 2004 Astron. Astrophys. 417, 325-332.

Karlický, M., Fárník, F., Mészárosová, H. 2002 Astron. Astrophys. 395, 677-683.

Khan, J.I. \& Aurass, H. 2002 Astron. Astrophys. 383, 1018-1031.

Khan, J.I. \& Hudson, H.S. 2000 Geophys. Res. Lett. 27, 1083-.

Klassen, A., Aurass, H., Klein, K.-L., Hofmann, A., and Mann, G. 1999 Astron. Astrophys. 343, 287-296.

Klassen, A., Pohjolainen, S., and Klein, K.-L. 2003 Solar Phys. 218, 197-210.

Klein, K-L., Khan, J.I., Vilmer, N., Delouis, J-M., and Aurass, H. 1999 Astron. Astrophys. 346, L53-L56.

Kosugi, T., Ishiguro, M. \& Shibasaki, K. 1986 Publ. Astron. Soc. Japan 38, 1-11.

Krucker, S. Benz, A.O., Bastian, T.S., and Acton, L.W. 1997 Astrophys. J. 488, 499-.

Kundu, M.R., Garaimov, V.I., White, S.M., and Krucker S. 2004 Astrophys. J. 600, 1052-1060.

Kundu, M.R., Nindos, A. \& Grechnev, V.V. 2004 Astron. Astrophys. 420, 351-359.

Lee, J., Gallagher, P.T., Gary, D.E., Nita, G.M., Choe, G.S. et al. 2003 Astrophys. J. 585, $524-535$.

Lehtinen, N.J., Pohjolainen, S., Karlicky, M., Aurass, H., and Otruba, W. 2004 Astron. Astrophys., submitted.

Liu, C., Qiu, J., Gary, D. E., Krucker, S., Wang, H. 2004 Astrophys. J. 604, 442-448.

Maia, D., Pick, M., Vourlidas, A., and Howard, R. 2000 Astrophys. J. 528, L49-L51.

Mancuso, S. \& Raymond, J.C. 2004 Astron. Astrophys. 413, 363-371.

Mandrini, C.H., Pohjolainen, S., Dasso, S., Green, L.M., Démoulin, P. et al. 2004 Astron. Astrophys. submitted.

Moran, T., Gopalswamy, N., Dammasch, I.E., and Wilhelm, K. 2001 Astron. Astrophys. 378, $1037-1045$.

Narukage, N., Hudson, H.S., Morimoto, T., Akiyama, S., Kitai, R., et al. 2002 Astrophys. J. 572, L109-112.

Nindos, A., Kundu, M.R., White, S.M., Gary, D.E., Shibasaki, K., and Dere, K.P. 1999 Astrophys. J. 527, 415-425.

Pohjolainen, S. 2003 Solar Phys. 213, 319-339.

Pohjolainen, S., Maia, D., Pick, M., Vilmer, N., Khan, J.I., et al. 2001 Astrophys. J. 556, 421-431.

Pohjolainen, S., Portier-Fozzani, F., and Ragaigne, D. 2000 Astron. Astrophys. Suppl. Ser. 143, 227-263.

Pohjolainen S., Vilmer N., Khan J.I., and Hillaris A.E. 2004 Astron. Astrophys. submitted.

Riehokainen, A., Urpo, S., Valtaoja, E., Makarov, V.I., Makarova, L.V., and Tlatov, A.G. 2001 Astron. Astrophys. 366, 676-685.

Selhorst, C.L., Silva, A.V.R., Costa, J.E.R., and Shibasaki, K. 2003 Astron. Astrophys. 401, $1143-1150$.

Shibasaki, K. 1998 In Synoptic Solar Physics (ed. K.S. Balasubramaniam, J. Harvey, and D. Rabin). ASP Conf. Ser., 140, 373-385.

Torsti, J., Kocharov, L., Laivola, J., Chertok, I., Thompson, B.J. 2003 Solar Phys. 214, 177-193.

Torsti, J., Kocharov, L., Laivola, J., Pohjolainen, S, Plunkett, S.P. et al. 2002 Solar Phys. 205, 123-147.

White, S.M., Krucker, S., Shibasaki, K., Yokoyama, T., Shimojo, M., and Kundu, M.R. 2003 Astrophys. J. 595, L111-114. 